\title{
Structural and Functional Features of Modified Heat-Stable Toxins Produced by Enteropathogenic Klebsiella Cells
}

\author{
FABIO ALBANO, MICHAEL R. THOMPSON, STEFANIA ORRÙ, ANDREA SCALONI, \\ ANTONELLA MUSETTA, PIERO PUCCI, AND ALFREDO GUARINO \\ Department of Pediatrics [F.A., M.R.T., A.M., A.G.], International Mass Spectometry Facilities Center, \\ CNR [S.O., A.S., P.P.]; Department of Organic and Biologic Chemistry, University "Federico II", \\ 80131 Naples, Italy [S.O., P.P.]; and the Unesco Center for Emerging and Re-emerging \\ Infections, Naples, Italy [A.G.]
}

\begin{abstract}
Heat-stable enterotoxins (STs) are 18- or 19-amino acid peptides (STa or ST1) produced by enteropathogenic bacteria with small differences in their amino acid sequence and a highly conserved carboxy terminus. All STs contain a core of three disulfide bridges whose integrity is believed to be necessary for full biologic activity. We previously reported that strains of Klebsiella pneumoniae transformed by the plasmid pSLM004 produce a modified toxin not recognized by MAb raised against genuine Escherichia coli ST. Investigation of the chemical structure of the modified toxins revealed that three new toxins were present. These were purified to homogeneity by a series of sequential chromatography on reverse-phase columns using guanylate cyclase to monitor the enterotoxic activity during purification procedures. The sequence of the modified toxins was obtained by a combination of Edman degradation and mass spectrometry, showing that they are proteolytically processed forms of E. coli ST1b. In particular, toxin A-2 lacks the cysteine at position 18 and then is not able to form the disulfide bridge cysteine-10-cysteine-18. All three toxins showed the ability to
\end{abstract}

\section{ABSTRACT}

stimulate guanylate cyclase and to elicit chloride secretion in Caco-2 cell monolayers mounted in Ussing chambers. Toxin A-1 and toxin $\mathrm{B}$ demonstrated greatly reduced immunoreactivity whereas toxin A-2 was not recognized at all in the ELISA. It is likely that the three modified toxins were generated by Klebsiella specific proteolytic processing of the original pretoxin. These results have important implications for the diagnosis and prevention of heat-stable toxin-induced diarrhea. (Pediatr Res 48: 685-690, 2000)

TFA, trifluoroacetic acid

\section{Abbreviations}

PD, transepithelial potential difference

Isc, short-circuit current

$\mathbf{G}$, tissue conductance

ESMS, electrospray mass spectrometry

ST, heat-stable enterotoxin

Cys, cysteine

Asn, asparagine
Escherichia coli producing STs are among the most frequent agents of diarrhea worldwide $(1,2)$. The STs constitute a family of low molecular mass peptide toxins, named STa or ST1, produced by a number of pathogenic bacteria (3). Various forms of these toxins exist, differing in their amino acid sequence but all containing a cysteine-rich core that is essential for the full exploitation of their biologic activity $(4,5)$. Within the core, the cysteine residues are all arranged in disulfide bridges whose integrity seems to be an absolute structural requirement for maintaining the functional conformation of the toxins $(5,6)$.

Received November 9, 1999; accepted June 21, 2000.

Correspondence: Dr. Alfredo Guarino, Department of Pediatrics, University "Federico II," Via S. Pansini 5, 80131 Naples, Italy.

Supported by a grant from Ministero della Sanità, Istituto Superiore di Sanità, AIDS research project 1998, Programme 50B.34.
Two prototypes of ST1 have been characterized, displaying minor differences both in the gene and in the amino acid sequence (7). The first toxin, ST1a or STp, is an 18-amino acid peptide, whereas ST1b or STh consists of 19 residues. Both ST1a and ST1b induce diarrhea through a multistep process involving the binding of the toxin to guanylate cyclase-C located on the intestinal brush border membranes, causing an increase in the intracellular concentration of cGMP and chloride secretion $(8,9)$.

The production of an ST-like toxin by Klebsiella pneumoniae strain AL55 isolated from a child affected by diarrhea has previously been reported (10). The supernatant of the Klebsiella cell culture contained an enterotoxic activity similar to that classically produced by ST-positive E. coli in that it induced secretion in the suckling mouse assay and was heat resistant but sensitive to 2-mercaptoethanol (10). Subse- 
quently, the Klebsiella AL55 strain lost its ability to express the enterotoxic activity and was then transformed both by insertion of the pSLM004 plasmid, which encodes for the $E$. coli ST1b, and through conjugation experiments using STpositive E. coli as donor (11). In both cases, the transformed cells showed the ability to produce an unexpected modified form of ST that retained biologic activity in the suckling mouse assay but was not recognized by antibody raised against both E. coli ST1a and ST1b in the ELISA tests.

This paper describes the chemical structure and functional correlates of the modified toxin. The protein was expressed in transformed Klebsiella cells, and activation of guanylate cyclase was used to monitor enterotoxic activity during the purification procedures. Three different toxins were detected and purified to homogeneity. Their structure was elucidated by a combination of Edman degradation and mass spectrometric methodologies, showing that they are proteolytically processed forms of E. coli ST1b. Functional and immunologic data on the homogeneous toxins were also obtained.

\section{METHODS}

Transformation experiments were performed as described (12), by incubating competent original Klebsiella AL55 cells (10), cured of the original plasmid, with the plasmid pSLM004, carrying a gene encoding for 19-amino acid ST1 and a gene for ampicillin resistance (13). Transformed cells were selected by their growth on LB agar supplemented with $200 \mu \mathrm{g} / \mathrm{mL}$ of ampicillin, to which Klebsiella AL55 was originally sensitive. Transformed Klebsiella AL55 was grown in L-broth supplemented with $200 \mu \mathrm{g} / \mathrm{mL}$ of ampicillin for toxin production. Briefly, $5 \mathrm{~mL}$ of an overnight starter culture was used to inoculate $500 \mathrm{~mL}$ of medium. The culture was grown for $18 \mathrm{~h}$ at $37^{\circ} \mathrm{C}$ with shaking, and then organisms were removed by centrifugation. Culture supernatant was sterilized by filtering it through $0.45-\mu \mathrm{m}$ pore size membranes. To investigate the similarities and differences between Klebsiella toxin and ST1b, E. coli HB101 was transformed with plasmid pSLM004 and used as a positive control.

Purification of Toxins. E. coli ST1b and the Klebsiella toxins were partially purified from the culture filtrate by Amberlite XAD and Sep-Pack chromatography as previously described (14). Toxins were obtained in a homogeneous form by three HPLC chromatographic steps performed on a $\mu$ Bondapack C18 column $(30 \times 0.39 \mathrm{~cm}$, Waters, Milford, MA, U.S.A.) using $0.1 \%$ TFA (solvent A) and $0.1 \%$ TFA in acetonitrile (solvent B) as the elution system at a flow rate of 1 $\mathrm{mL} / \mathrm{min}$. The partially purified toxins were first eluted by means of a linear gradient of solvent B from 15 to $24 \%$ in 10 $\mathrm{min}$ and then to $30 \%$ in $40 \mathrm{~min}$. The active fractions were dried down, dissolved in $10 \mathrm{mM}$ ammonium acetate, $\mathrm{pH} 5.8$, and chromatographed again using a linear gradient of solvent B from 12 to $18 \%$ in $10 \mathrm{~min}$ and then to $24 \%$ in $40 \mathrm{~min}$. Finally, the last chromatographic step was performed by a slightly different gradient of solvent B from 15 to $20 \%$ in 10 min and then to $25 \%$ in $40 \mathrm{~min}$.

Enzymatic and immunologic assays. Guanylate cyclase assay was performed by a modification of the method of Wald- man et al. (15). The activity of guanylate cyclase was expressed as picomoles of cGMP formed per minute per milligram of protein.

ELISA tests were performed on crude extracts and homogeneous toxins by using MAb raised against pure E. coli ST1a toxin (16). These antibodies recognize both ST1a and ST1b toxins (17).

Ussing chamber studies. Caco-2 cells derived from human colonic adenocarcinoma differentiate as mature enterocytes and were used to investigate the enterotoxic effects of Klebsiella toxins. Caco-2 cell monolayers were grown on uncoated, nontransparent polycarbonate transwell filters $(0.4-\mu \mathrm{m}$ pore size, $24.5 \mathrm{~mm}$ diameter) at a concentration of $2 \times 10^{6}$ cells per filter and were mounted in Ussing chambers as described (18). $\mathrm{PD}$, Isc, and $\mathrm{G}$ were measured as described (19). An increase in PD indicates anion secretion, which is the classic effect of enterotoxins, whereas an increase in $\mathrm{G}$ indicates a cytotoxic effect. Electrical variables were recorded before and after the addition of the toxin preparations. Cell viability was determined at the end of each experiment by the electrical response to serosal addition of $5 \mathrm{mM}$ of theophylline.

Structural characterization of toxins. HPLC-purified toxins were reduced in $0.25 \mathrm{M}$ Tris-HCl, $\mathrm{pH} 8.5$, containing $1.25 \mathrm{mM}$ EDTA and $6 \mathrm{M}$ guanidinium chloride, with a 10:1 molar excess of DTT over the sulphydryl (SH) groups, for $2 \mathrm{~h}$ at $37^{\circ} \mathrm{C}$ under nitrogen atmosphere. Alkylation of cysteine residues was performed in the same buffer, with a 5:1 molar excess of iodoacetic acid over the total $\mathrm{SH}$ groups, for $30 \mathrm{~min}$ at room temperature in the dark under nitrogen atmosphere. Alkylated proteins were desalted by reverse-phase HPLC on a Vydac C18 column $(25 \times 0.46 \mathrm{~cm}, 5 \mu \mathrm{m}$, The Separation Group, Hesperia, CA, U.S.A.) using $0.1 \%$ TFA (solvent A) and $0.07 \%$ TFA in $95 \%$ acetonitrile (solvent $\mathrm{B}$ ) by means of a linear gradient from 15 to $95 \%$ solvent B in $10 \mathrm{~min}$.

The amino acid sequence of the carboxymethylated toxins was determined by using an Applied Biosystem 477A pulsedliquid protein sequencer equipped with an Applied Biosystem 120A HPLC apparatus for phenylthiohydantoin amino acid identification (Perkin Elmer, Foster City, CA, U.S.A.).

ESMS analyses were performed using a BIO-Q triple quadrupole mass spectrometer equipped with an electrospray ion source (Micromass, Manchester, U.K.). In a typical experiment, $10 \mu \mathrm{L}$ of each toxin peak was directly injected into the ion source via loop injection, at a flow rate of $10 \mu \mathrm{L} / \mathrm{min}$. Spectra were recorded by scanning the first quadrupole at 10 s/scan. Data were acquired and elaborated by the MassLynx software (Micromass). Mass scale calibration was performed by means of multiply charged ions from a separate injection of horse heart myoglobin (average molecular mass, 16,951.5 D).

\section{RESULTS}

Both E. coli and Klebsiella AL55 strains were negative in both the ELISA test and guanylate cyclase assay. When E. coli strain HB101 and Klebsiella AL55 cells were transformed with the pSLM004 plasmid, both culture supernatants showed cross-immunoreactivity with MAb raised against $E$. coli ST1a and the ability to stimulate guanylate cyclase activity. A small- 
scale purification procedure essentially consisting of three HPLC chromatographic steps yielded sufficient amounts of the E. coli toxin, which was rapidly identified as true ST1b by ESMS (see below). A single peak of ST-cross immunoreacting material that corresponded to the biologically active fraction was observed after each chromatographic separation. Figure $1 A$ shows the final HPLC profile of pure ST1b; approximately $200 \mu \mathrm{g}$ of toxin was purified from $500 \mathrm{~mL}$ of transformed $E$. coli HB101 supernatant.

On the contrary, when the concentration of the toxin present in the supernatant of Klebsiella AL55 was quantitated either by ELISA or by the guanylate cyclase assay, substantial discrepancy was observed. According to the guanylate cyclase doseresponse stimulation curve, ST concentration was increased 5 -fold compared with that estimated by the ELISA assay. Moreover, the HPLC profile of the crude culture supernatant showed two distinct peaks, indicated as fractions A and B, both displaying the peculiar biologic activity and immunoreactivity of ST1b, but showing different retention times compared with the E. coli toxin (Fig. 1B). Fraction B was homogeneous to further purification procedures and to mass spectral analysis, whereas the HPLC analysis of component A on a different



Figure 1. HPLC purification of E. coli and Klebsiella AL55 toxins. a, homogeneous ST1b from E. coli. $b$, crude culture supernatant of Klebsiella AL55 displaying the presence of two components, A and B, endowed with the peculiar biologic activity and immunoreactivity of ST1b. $c$, HPLC analysis of component $\mathrm{A}$ on a different chromatographic system, showing the occurrence of two different toxins, named A-1 and A-2. chromatographic system revealed the presence of two different species named A-1 and A-2 (Fig. 1C), which were eventually purified to homogeneity.

Structural characterization of the novel toxins. The putative E. coli ST1b and the three new toxins purified from transformed Klebsiella AL55 were analyzed by ESMS. The $E$. coli toxin showed a molecular mass of $2040.9 \pm 0.1 \mathrm{D}$, almost coincident with the expected value for true ST1b (2041.3 D), thus confirming the identification. The ESMS spectra of toxins A-1, A-2, and B are shown in Figure 2; component B displayed a mass value of $1927.2 \pm 0.2 \mathrm{D}$, approximately $114 \mathrm{D}$ lower than that of ST1b, suggesting that the N-terminal Asn residue was missing. The molecular masses of toxins A-1 and A-2 were measured as $1476.1 \pm 0.2 \mathrm{D}$ and $1504.3 \pm 0.5 \mathrm{D}$, respectively, and were tentatively assigned to differently truncated forms of ST1b.

The three toxins were then reduced and carboxymethylated to block the cysteine residues and submitted to automated Edman degradation, yielding the amino acid sequences reported in Table 1, in which the primary structure of ST1b is also shown for comparison. This analysis confirmed that all the toxins purified from transformed Klebsiella cells were generated by different proteolytic processing of the original E. coli ST1b. In particular, toxin B lacks the N-terminal Asn residue, as predicted on the basis of the ESMS data, whereas toxin A-1 represents the N-terminal-truncated species 6-19, in which the

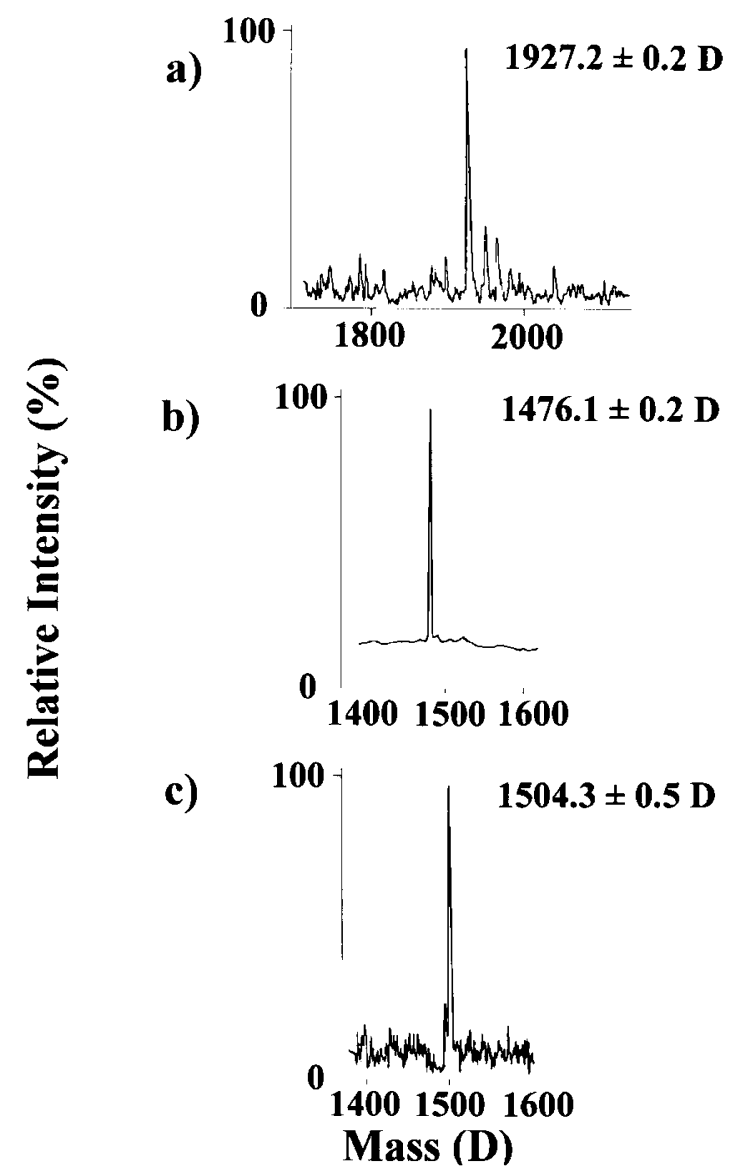

Figure 2. Transformed ESMS of the new toxins from Klebsiella AL55, toxins A-1 (a), A-2 (b), and B (c). The measured molecular masses are indicated. 
Table 1. Amino acid sequence of the three new toxins derived from engineered Klebsiella pneumonia AL55 strain

\begin{tabular}{|c|c|c|c|c|}
\hline & 1 & 5 & 10 & 15 \\
\hline ST1b & \multirow{2}{*}{\multicolumn{4}{|c|}{$\begin{array}{l}\text { Asn-Ser-Ser-Asn-Tyr-Cys-Cys-Glu-Leu-Cys-Cys-Asn-Pro-Ala-Cys-Thr-Gly-Cys-Tyr } \\
\text { Cys-Cys-Glu-Leu-Cys-Cys-Asn-Pro-Ala-Cys-Thr-Gly-Cys-Tyr } \\
\text { Ser-Ser-Asn-Tyr-Cys-Cys-Glu-Leu-Cys-Cys-Asn-Pro-Ala-Cys-Thr-Gly-Cys-Tyr } \\
\text { Ser-Ser-Asn-Tyr-Cys-Cys-Glu-Leu-Cys-Cys-Asn-Pro-Ala-Cys }\end{array}$}} \\
\hline Toxin B & & & & \\
\hline
\end{tabular}

The primary structure of E. coli $\mathrm{ST} 1 \mathrm{~b}$ is also included for comparison.

first five residues have been removed. Toxin A-2 corresponds to the C-terminal-truncated form $2-15$, in which the last four residues were missing. It is interesting to note that the latter protein lacks the cysteine residue at position 18 , and thus it is not able to form the disulfide bridge Cys10-Cys18 (6).

Functional and immunologic studies. The biologic activity of the three Klebsiella toxins was tested by dose-response experiments of guanylate cyclase activation using $E$. coli $\mathrm{ST} 1 \mathrm{~b}$ as reference. All three toxins showed the ability to stimulate enzymatic activity. The dose-response curves of each of the three Klebsiella toxins elicited an increase in cGMP concentration that had a pattern similar to that observed with E. coli ST1b. However, their potency was reduced compared with the standard E. coli toxin, as judged by the magnitude of cGMP response (Fig. 3). The biologic activities of ST1b and of Klebsiella toxins were also evaluated in Caco-2 cells mounted in Ussing chambers to assess the secretory potency of the toxins. As expected, Klebsiella toxin generated an increase in Isc entirely related to a modification of PD inasmuch as no modification of $\mathrm{G}$ was recorded. Figure 4 shows that ST1b and toxin B generated a similar pattern of Isc response. However, the peak intensity elicited by toxin B was lower than that induced by ST1b, in keeping with the concept that guanylate cyclase stimulation and intestinal secretion are coupled processes. Preliminary experiments showed that Klebsiella toxins A-1 and A-2 also generated an increase in Isc, which was of reduced magnitude compared with both $E$. coli $\mathrm{ST} 1 \mathrm{~b}$ and Klebsiella toxin B (data not shown).

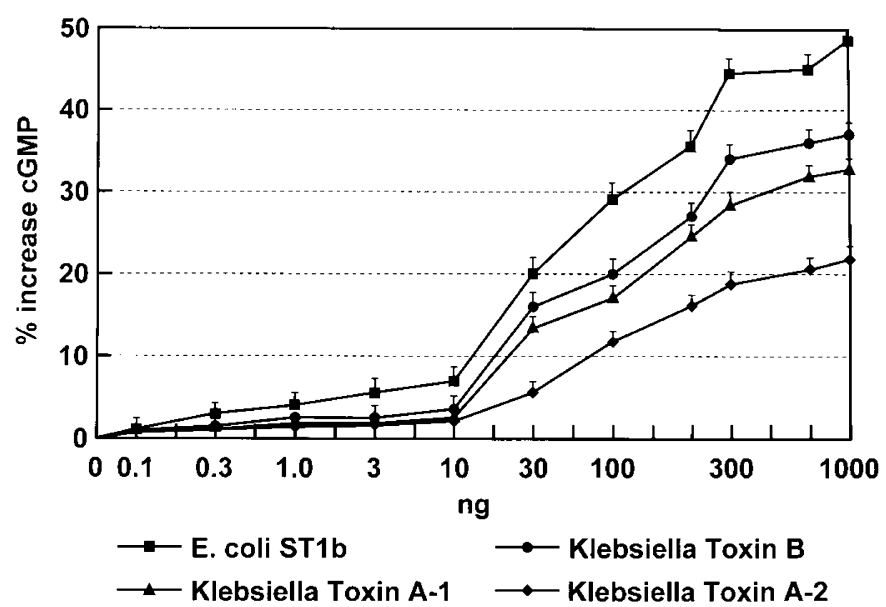

Figure 3. Dose-dependent activation of guanylate cyclase by E. coli ST1b and by Klebsiella toxins A-1, A-2, and B. The kinetics of guanylate cyclase activation in response to E. coli $\mathrm{ST} 1 \mathrm{~b}$ and to Klebsiella toxins A-1, A-2, and $\mathrm{B}$ were similar, indicating similar biologic effects of the toxin isoforms. All toxin isoforms induced a significant increase in cGMP concentrations. However, E. coli ST1b was slightly but significantly more potent than Klebsiella toxins. Data are mean $\pm \mathrm{SE}$ of four separate experiments.

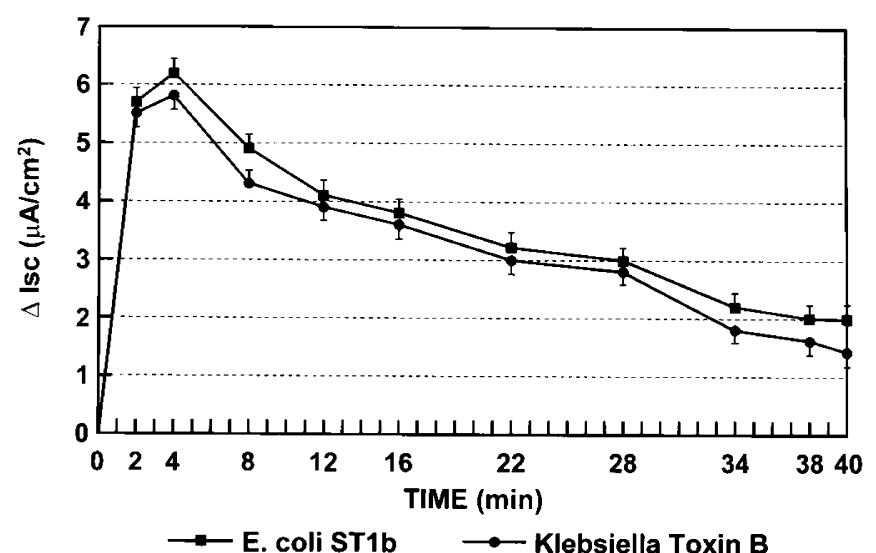

Figure 4. Enterotoxic effects of Klebsiella toxin B and of E. coli ST1b as judged by the intensity of Isc in Caco-2 cells. Klebsiella toxin B and E. coli ST1b showed a similar time course of the enterotoxic activity. Data are mean $\pm \mathrm{SE}$ of four separate experiments.

ELISA assays were performed on the purified new toxins by using MAb raised against $E$. coli ST1a, and the immunologic data are summarized in Table 2. Although the antibodies were able to recognize both toxins $\mathrm{A}-1$ and $\mathrm{B}$, these proteins displayed a greatly reduced immunoreactivity compared with native $E$. coli toxins. In particular, toxin A-1 originated a poor response close to the detection limit of the assay under the conditions used that could not be quantitated. Finally, toxin A-2 was not recognized at all by the antibodies.

\section{DISCUSSION}

The genes encoding for ST toxins are usually located in plasmids, readily allowing interspecies transfer of transposons that can confer toxigenicity to previously nontoxigenic microorganisms both in vivo and in vitro. The K. pneumoniae AL55 strain used in this study was originally isolated from a child with acute secretory diarrhea (10). The child's stools also contained an ST-producing strain of E. coli, suggesting that the plasmid coding for ST toxins might have been transferred from

Table 2. ELISAs performed on three new toxins derived from Klebsiella pneumonia AL55 strain using E. coli STla and ST1b proteins as reference

\begin{tabular}{lccc}
\hline \multicolumn{1}{c}{ Toxin } & $\begin{array}{c}\text { Quantity (pg) } \\
\text { (amino acid analysis) }\end{array}$ & $\begin{array}{c}\text { Quantity (pg) } \\
\text { (ELISA) }\end{array}$ & $\begin{array}{c}\text { Identification } \\
(\%)\end{array}$ \\
\hline ST1a & 1000 & 1000 & 100 \\
ST1b & 1000 & 600 & 60 \\
Toxin B & 1000 & 250 & 25 \\
Toxin A-1 & 1000 & positive & ND \\
Toxin A-2 & 1000 & - & - \\
\hline
\end{tabular}

$\mathrm{ND}$, not determined

The concentration of the toxin solution was determined by amino acid analysis. 
E. coli to Klebsiella in vivo. To test this hypothesis, a Klebsiella AL55 strain unable to produce ST toxins was transformed in vitro with an ST-encoding plasmid from E. coli. The supernatant from engineered Klebsiella cells showed positive response in both the suckling mouse assay and the activation of guanylate cyclase but did not display cross-immunoreactivity with MAb raised against pure $E$. coli ST1a under the conditions used to screen for enterotoxigenic E. coli strains (11).

These unexpected functional features were shown to be related to the expression of three different enterotoxins that were then purified to homogeneity and structurally characterized by a combination of mass spectrometric and automated Edman degradation methodologies. The three novel toxins produced by Klebsiella were identified as both $\mathrm{N}$ - and Cterminal proteolytically processed forms of the E. coli $\mathrm{ST} 1 \mathrm{~b}$ protein. Under the conditions used, no production of the native 19-amino acid ST1b toxin was observed. All three toxins lack the first Asn residue, with toxin A-1 being further shortened at the $\mathrm{N}$-terminus where four additional residues are missing. In contrast, toxin A-2 represents a C-terminal-truncated species of ST1b that lacks the last four residues, including Cys18.

The absence of this cysteine residue makes toxin A-2 unable to form the Cys10-Cys18 disulfide bridge. All ST toxins contain a structural core stabilized by the three disulfides Cys6-Cys11, Cys7-Cys15, and Cys10-Cys18, which is thought to be a crucial element to elicit full biologic activity. However, two distinct ST-like peptides that contain only two rather than three disulfide bridges have also been described. Both guanylin, an endogenous peptide extracted from the rat small intestine (20) and EAST1, an enterotoxin produced by enteroaggregative $E$. coli (21), lack the disulfide bridge involving Cys6 and Cys11. Yet both peptides displayed binding activity to the guanylate cyclase receptor and subsequent secretory activity similar to the other ST toxins. As mentioned above, toxin A-2 produced by Klebsiella strain lacks the disulfide bridge Cys10-Cys18, but it is still able to stimulate cGMP production. These findings might suggest that the absolute structural requirement for the toxin-receptor interaction consists in the presence of two disulfide bridges, one of which must be the Cys7-Cys15 disulfide bond. This observation is strongly supported by the key role of this disulfide bridge in EAST1 as inferred by site-directed mutagenesis experiments in which Cys7 had been replaced with alanine, leading to the complete abolishment of guanylate cyclase activation (6).

The novel toxins produced by Klebsiella were able to stimulate cGMP production with dose-response kinetics similar to those observed with E. coli ST1b. Moreover, modified Klebsiella toxins displayed a comparable activity in inducing $\mathrm{Cl}^{-}$ secretion in Caco-2 monolayers mounted on semipermeable supports in Ussing chambers. Both toxins B and A-1 demonstrated greatly reduced immunoreactivity in the MAb ELISA test, suggesting that the first Asn residue and, in general, the $\mathrm{N}$-terminal portion may play a role in the recognition of ST toxins by the antibodies. However, the most intriguing result obtained in the ELISA assays was that toxin A-2 was not recognized by the MAb that had previously been used for surveillance studies on the frequency of ST1a-producing $E$. coli. Indeed in previous epidemiologic studies, four of 31 enterotoxigenic E. coli strains were found to be positive in the suckling mouse assay but negative in the ELISA (2). The hypothesis of a change in primary structure was raised, and it has now been confirmed. The inability of the ELISA tests to recognize the enterotoxin might be associated with either the absence of the C-terminal tyrosine residue or the lack of the structural elements at the C-terminus because of the absence of the Cys10-Cys18 disulfide bridge. Therefore, identification of modified toxins in culture supernatants by ELISA assay can yield false-negative results in epidemiologic studies leading to underestimation of ST-producing microorganisms.

At present, the prevalence of Klebsiella and of E. coli strains able to produce multiple forms of ST1a and ST1b toxins is not known, nor is it a putative modification mechanism. The E. coli ST toxins are small, compact, and stable proteins, very resistant to proteases, and yet Klebsiella AL55 should contain one or more enzymatic activities that can digest ST toxins or their precursors. The possibility that ELISA-positive enterotoxigenic strains can also produce high quantities of modified toxins such as Klebsiella AL55 and therefore be even more aggressive cannot be ruled out. A prevalence study should be performed to understand whether other Klebsiella strains or other bacterial species associated with secretory diarrhea exist and how these strains are capable of modifying toxins. It is therefore important to develop specific reagents to identify all forms of STs because at present, surveillance studies are conducted with reagents that might not be suitable for this purpose.

The failure of antibodies to recognize at least one of the variant toxins raises the problem of the protective efficacy of vaccines prepared against STs (22). Further characterization of multiple phenotypes of ST toxins is important to assess the efficacy of vaccines. A vaccine effective for $E$. coli ST1a and ST1b could be not as efficient against modified STs.

\section{REFERENCES}

1. Black RE, Brown KH, Becker S, Abdul Alim ARM, Huq I 1982 Longitudinal studies of infectious diseases and physical growth of children in rural Bangladesh: II. Incidence of diarrhea and association with known pathogens. Am J Epidemiol $115: 315-324$

2. Guarino A, Alessio M, Tarallo L, Fontana M, Iacono G, Gobio Casali L, Guandalini S 1989 Heat-stable enterotoxin produced by Escherichia coli in acute diarrhoea. Arch Dis Child 64:808-813

3. Cohen MB, Giannella RA 1995 Enterotoxigenic E. coli. In: Blaser MJ, Smith PD, Ravdin JI, Greenberg HB (eds) Infections of the Gastrointestinal Tract. Raven Press, New York, pp 691-707

4. Aimoto S, Watanabe H, Ikemura H, Shimonishi Y, Takeda T, Takeda Y, Miwatani T 1983 Chemical synthesis of a highly potent and heat-stable analog of an enterotoxin produced by a human strain of enterotoxigenic Escherichia coli. Biochem Biophys Res Commun 112:320-326

5. Gariepy J, Judd AK, Schoolnik GK 1987 Importance of disulfide bridges in the structure and activity of Escherichia coli enterotoxin ST1b. Proc Natl Acad Sci USA 84:8907-8911

6. Fasano A 1998 Cellular microbiology: how enteric pathogens socialize with their intestinal host. J Pediatr Gastroenterol Nutr 26:520-532

7. Thompson MR 1987 Escherichia coli heat-stable enterotoxins and their receptors. Pathol Immunopathol Res 6:279-286

8. Field M, Graf LH, Laird WJ, Smith PL 1978 Heat-stable enterotoxin of Escherichia coli: in vitro effects on guanylate cyclase activity, cyclic GMP concentration, and ion transport in small intestine. Proc Natl Acad Sci USA 75:2800-2804

9. Giannella RA, Luttrell M, Thompson MR 1983 Binding of Escherichia coli heatstable enterotoxin to receptors on rat intestinal cells. Am J Physiol 245.G492-G498

10. Guarino A, Guandalini S, Alessio M, Gentile F, Tarallo L, Capano G, Migliavacca M, Rubino A 1989 Characteristics and mechanism of action of a heat-stable enterotoxin produced by Klebsiella pneumoniae from infants with secretory diarrhea. Pediatr Res 25:514-518 
11. Alessio M, Albano F, Tarallo L, Guarino A 1993 Interspecific plasmid transfer and modification of heat-stable enterotoxin expression by Klebsiella pneumoniae from infants with diarrhea. Pediatr Res 33:205-208

12. Cohen SN, Chang ACY, Hsu L 1972 Nonchromosomal antibiotic resistance in bacteria: genetic transformation of $E$. coli by R-factor DNA. Proc Natl Acad Sci USA 69:2110-2114

13. Moseley SL, Hardy JW, Huq MI, Echeverria P, Falkow S 1983 Isolation and nucleotide sequence determination of a gene encoding a heat-stable enterotoxin of Escherichia coli. Infect Immun 39:1167-1174

14. Guarino A, Giannella R, Thompson MR 1989 Citrobacter freundii produces an 18-amino-acid heat-stable enterotoxin identical to the 18 amino-acid Escherichia coli heat-stable enterotoxin (ST Ia). Infect Immun 57:649-652

15. Waldman SA, O'Hanley PD, Falkow S, Schoolnik G, Murad F 1984 A simple, sensitive and specific assay for heat-stable enterotoxin of Escherichia coli. J Infect Dis 149:83-89

16. Thompson MR, Brandwein H, Labine-Recke M, Giannella RA 1984 Simple and reliable enzyme-linked immunosorbent assay with monoclonal antibodies for detection of Escherichia coli enterotoxin. J Clin Microbiol 20:59-64

17. Thompson MR, Jordan RL, Luttrell MA, Brandwein H, Kaper JB, Levine MM, Giannella RA 1986 Blinded, two laboratory comparative analysis of Escherichia coli heat-stable enterotoxin production by using monoclonal antibody enzyme linked immunosorbent assay, radioimmunoassay, suckling mouse assay, and gene probes. J Clin Microbiol 24:753-758

18. Guarino A, Berni Canani R, Casola A, Pozio E, Russo R, Bruzzese E, Fontana M, Rubino A 1995 Human intestinal cryptosporidiosis: secretory diarrhea and enterotoxic activity in Caco-2 cells. J Infect Dis 171:976-983

19. Field M, Fromm D, McColl I 1971 Ion transport in rabbit ileal mucosa. I. Na and Cl fluxes and short circuit current. Am J Physiol 87:1450-1455

20. Currie MG, Fok KF, Kato J, Moore RJ, Hamra FK, Duffin KL, Smith CE 1992 Guanylin: an endogenous activator of intestinal guanylate cyclase. Proc Natl Acad Sci USA 89:947-951

21. Savarino SJ, Fasano A, Watson J, Martin BM, Levine MM, Guandalini S, Guerry P 1993 Enteroaggregative Escherichia coli heat-stable enterotoxin 1 represents a new subfamily of E. coli heat-stable toxin. Proc Natl Acad Sci USA 90:30933097

22. Anonymous $1999 \mathrm{New}$ frontiers in the development of vaccines against enterotoxinogenic (ETEC) and enterohaemorrhagic (EHEC) E. coli infections. Part I. Wkly Epidemiol Rec 74:98-101 